\title{
The $\mathbf{C}^{1} \Sigma^{+}$State of KLi Studied by Polarisation Labelling Spectroscopy Technique
}

\author{
A. Grochola ${ }^{a}$, P. KowalczyK ${ }^{a}$, W. JAstrzęBSki ${ }^{b}$, \\ P. CROZET ${ }^{c}$ AND A.J. Ross ${ }^{c}$ \\ ${ }^{a}$ Institute of Experimental Physics, Warsaw University \\ Hoża 69, 00-681 Warsaw, Poland \\ ${ }^{b}$ Institute of Physics, Polish Academy of Sciences \\ al. Lotników 32/46, 02-668 Warsaw, Poland \\ ${ }^{c}$ Laboratoire de Spectrométrie Ionique et Moléculaire \\ Université Lyon I et CNRS (UMR 5579), Bâtiment Alfred Kastler \\ Domaine Scientifique de la Doua, 69622 Villeurbanne Cedex, France
}

(Received July 12, 2002)

The polarisation labelling spectroscopy method is applied to study the $C^{1} \Sigma^{+} \leftarrow X^{1} \Sigma^{+}$band system of the KLi molecule. Rotationally resolved polarisation spectra are observed in the spectral range of $17150-20350 \mathrm{~cm}^{-1}$. A set of Dunham coefficients describes the $C^{1} \Sigma^{+}$state to $95 \%$ of its potential well depth, and the potential curve is constructed by the Rydberg-Klein-Rees procedure. The molecular parameters deduced from this work are compared with theoretical calculations.

PACS numbers: 33.20.Kf, 33.15.-e, 34.20.Cf

\section{Introduction}

Mixed alkali dimers are often considered as the prototypes of heteronuclear diatomics. Due to their simple electronic configuration accurate quantum mechanical calculations are feasible (see e.g. [1-3] and references therein), while the main absorption bands conveniently positioned in the visible region make them also accessible to laser spectroscopy experiments [4]. The KLi molecule is one of the least known heteronuclear alkali dimers. The principal difficulty in experimental studies of this molecule is that the strong band systems of $\mathrm{K}_{2}$ and $\mathrm{Li}_{2}$ cover much of the visible spectrum, thus prohibiting isolation of the KLi spectroscopic features. 
Some years ago, we developed in Warsaw a dual-temperature heat pipe oven specifically designed to optimise the production of mixed alkali vapours [5] and using this source, we engaged in a series of spectroscopic investigations of KLi. Our work began with the high resolution study of the $B^{1} \Pi \leftarrow X^{1} \Sigma^{+}$band system by Doppler-free polarisation spectroscopy method, in which we obtained highly precise molecular constants for the lowest vibrational levels of both electronic states involved [6-8]. The characterisation of the ground $X^{1} \Sigma^{+}$state has been completed up to the dissociation limit in a later Fourier transform spectroscopy experiment [9]. For further studies of excited electronic states of KLi we have employed the polarisation labelling spectroscopy technique. The advantage of this method lies in considerable simplification of molecular spectra, as the observed transitions are limited only to these originating from a few vibrational levels "labelled" in the ground state. The outcome of our work was a complete characterisation of the $B^{1} \Pi$ state, observation of the lower part of the $C^{1} \Sigma^{+}$state (up to the vibrational level $v=19$ ) and a thorough analysis of the interaction between the $B$ and $C$ states $[10,11]$.

This paper focuses on our new analysis of the $C^{1} \Sigma^{+}$state. The present experiment has evolved, and with its improved sensitivity, we have extended the range of levels observed in the KLi molecule. Extending the spectrum to higher frequency has allowed us to observe vibrational levels of the $C$ state up to $v=36$, thus nearly doubling the amount of information about the shape of its potential curve and trebling the number of spectral lines used in the analysis. The molecular constants of the $C^{1} \Sigma^{+}$state reported in this paper describe $95 \%$ of its potential well depth.

In the following sections we present the details of the experimental method and the results of the measurements.

\section{Experimental details}

To study the $C^{1} \Sigma^{+} \leftarrow X^{1} \Sigma^{+}$system, we employed the $V$-type optical-optical double resonance polarisation labelling spectroscopy technique with two independent pump and probe lasers. In our version of the method the probe laser had a fixed frequency and excited a few assigned molecular transitions, whereas the pump laser was tuned over an investigated spectrum.

The pump laser was a pulsed dye laser (Lumonics HD500 pumped by a XeCl excimer laser EX500) delivering pulses of $2 \mathrm{~mJ}$ energy, $8 \mathrm{~ns}$ duration and $0.1 \mathrm{~cm}^{-1}$ spectral width. With Coumarines 540A, 503 and 480 as laser dyes, we covered the spectral range of $17150-20350 \mathrm{~cm}^{-1}$. The absolute calibration of the wave number scale was achieved by recording the optogalvanic signal from argon- and neon-filled hollow cathode lamps. The linearity of the laser scan was additionally controlled with a fixed-length Fabry-Pérot interferometer providing frequency markers separated by $1 \mathrm{~cm}^{-1}$. The accuracy in determination of absolute wave numbers of 
molecular lines was better than $0.1 \mathrm{~cm}^{-1}$ (this is roughly twice the Doppler width of KLi molecules produced in the heat pipe source).

The weaker, home built probe dye laser (Littman-type design [12], spectral width about $1 \mathrm{~cm}^{-1}$, pumped synchronously by the same excimer laser), was operated with a Rhodamine $6 \mathrm{G} / 110$ mixture, and was set at a fixed wavelength resonant with a few rovibronic transitions in the $B^{1} \Pi-X^{1} \Sigma^{+}$system of KLi, known from our previous high resolution studies [7, 8], thus labelling selected rovibrational levels in the ground state. For permanent control of the probe laser frequency during the measurements, we have chosen eight fixed wavelengths coinciding with strong optogalvanic lines of argon. Table I presents a summary of all the identified labelling transitions induced by the probe laser, which gave rise to the polarisation spectra.

The KLi molecules were prepared in a dual-temperature heat pipe oven in the presence of 4 torr of helium as a buffer gas. In short, the oven consists of two conventional heat pipes working on potassium, separated by a zone where metallic lithium is heated independently. At properly chosen working temperatures (in this experiment about $500^{\circ} \mathrm{C}$ in the centre and $280^{\circ} \mathrm{C}$ outside), potassium vapour penetrates the central zone and mixes with lithium vapour, giving favourable conditions for formation of $\mathrm{KLi}$.

The co-propagating pump and probe laser beams intersected in the central part of the oven at an angle of about $10 \mathrm{mrad}$ giving an interaction length of $c a .10 \mathrm{~cm}$. The pump beam could be polarised either circularly or linearly; after passing the KLi vapour it was removed by a beam stopper. Crossed polarisers were placed in the path of the probe beam on either side of the heat pipe. At frequencies corresponding to transitions induced by the pump beam which happen to originate from the same levels as the probe transitions, the probe beam was subjected to optical anisotropy generated in the molecular sample by the polarised pump beam. At these frequencies, the probe light changed its original polarisation and passed partially through the second polariser. Therefore the information about the excitation spectrum of KLi was contained in the transmitted intensity of the probe light. It follows from the general principles of polarisation spectroscopy that for a circularly polarised pump beam, only transitions originating from the levels labelled via $P$ or $R$ lines show up in the polarisation spectra, whereas in case of a linearly polarised pump beam, the spectra are dominated by transitions labelled via $Q$ lines [13]. These rules led to additional simplification of the observed spectra. For obvious reasons, in the applied detection method it was crucial to equip the heat pipe oven with windows of particularly low birefringence. We used individually selected fused silica windows whose residual birefringence was additionally compensated by gentle squeezing of the windows with adjustable screws. With the crossed polarisers and the pump beam off, an extinction of the probe beam better than $10^{-7}$ was obtained. 
TABLE I

Transitions in the $B^{1} \Pi \leftarrow \leftarrow X^{1} \Sigma^{+}$band system of ${ }^{39} \mathrm{~K}^{7} \mathrm{Li}$ excited by the probe (labelling) laser stabilised on atomic ArI lines.

\begin{tabular}{|c|c|}
\hline Probe laser line & Labelled transition $B\left(v^{\prime}, J^{\prime}\right)-X\left(v^{\prime \prime}, J^{\prime \prime}\right)$ \\
\hline $565.91 \mathrm{~nm}$ & $\begin{aligned}\left(1, J^{\prime}\right)-\left(0, J^{\prime \prime}\right)\left[J^{\prime \prime}\right. & \left.=2-15, J^{\prime}=J^{\prime \prime}+1\right] \\
& (2,43)-(0,43) \\
& (2,44)-(0,44) \\
& (2,47)-(0,46) \\
& (3,53)-(0,54) \\
& (3,56)-(0,56)\end{aligned}$ \\
\hline $568.19 \mathrm{~nm}$ & $\begin{array}{l}(1,30)-(0,31) \\
(1,31)-(0,32) \\
(1,32)-(0,33) \\
(1,39)-(0,38) \\
(1,40)-(0,39) \\
(1,41)-(0,40) \\
(2,50)-(0,51)\end{array}$ \\
\hline $568.99 \mathrm{~nm}$ & $\begin{array}{l}(1,36)-(0,37) \\
(5,40)-(2,39) \\
(1,40)-(0,40) \\
(1,41)-(0,41) \\
(2,57)-(0,57) \\
\end{array}$ \\
\hline $577.21 \mathrm{~nm}$ & $\begin{array}{c}(0,5)-(1,6) \\
(0,9)-(1,9) \\
(0,15)-(1,14)\end{array}$ \\
\hline $580.21 \mathrm{~nm}$ & $\begin{array}{l}(0,38)-(1,39) \\
(0,42)-(1,42) \\
(0,43)-(1,43) \\
\end{array}$ \\
\hline $583.43 \mathrm{~nm}$ & $\begin{array}{l}(2,19)-(3,20) \\
(2,23)-(3,23) \\
(3,50)-(3,49) \\
(0,60)-(1,60) \\
(0,65)-(1,64) \\
\end{array}$ \\
\hline $588.26 \mathrm{~nm}$ & $(0,45)-(2,46)$ \\
\hline $591.21 \mathrm{~nm}$ & $\begin{array}{l}(0,15)-(3,14) \\
(2,27)-(4,28) \\
(0,64)-(2,64)\end{array}$ \\
\hline
\end{tabular}


The residual part of the probe beam transmitted through the second polariser was directed to a $0.3 \mathrm{~m}$ monochromator centred at the probe wavelength and onto the photocathode (S-20 spectral response) of a photomultiplier tube connected to the boxcar averager (Stanford Research Systems, SR250). The polarisation spectra were stored in a computer, which also recorded the reference optogalvanic spectra together with the frequency markers of the etalon. With these input data the wave number of each line was measured with an absolute accuracy better than $0.1 \mathrm{~cm}^{-1}$. The same computer was used simultaneously to trigger the excimer laser and to control the tuning of the pump dye laser.

\section{Results and analysis}

Figure 1 shows a part of the observed spectrum representative for the region, where the $C^{1} \Sigma^{+} \leftarrow X^{1} \Sigma^{+}$band system was not overlapped by other spectral features of KLi. The analysis of the $C-X$ system was rather straightforward in cases when only a few $v^{\prime}$-progressions were present in a given polarisation spectrum. The assigned lines provided then preliminary molecular constants of the $C$ state

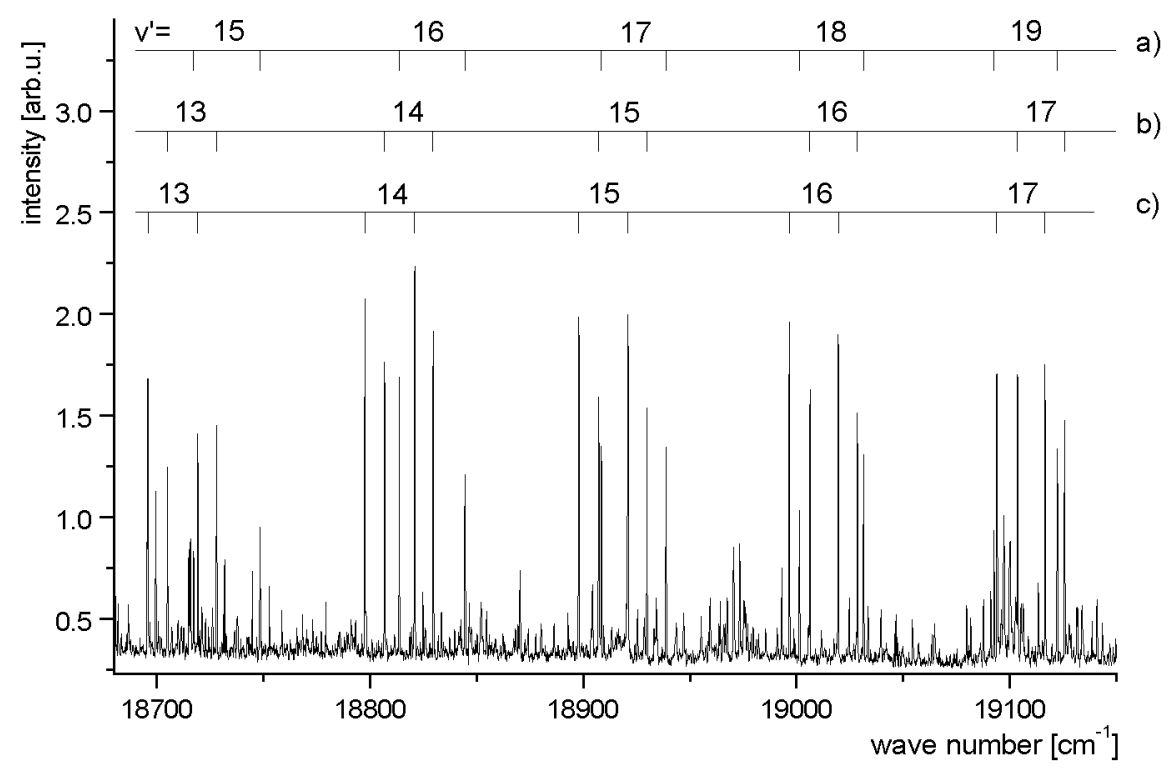

Fig. 1. A portion of the polarisation spectrum of KLi observed with linearly polarised pump laser beam. The assigned progressions (a)-(c) correspond to transitions $C^{1} \Sigma^{+}-X^{1} \Sigma^{+}$from the ground state levels (a) $v^{\prime \prime}=0, J^{\prime \prime}=57$, (b) $v^{\prime \prime}=0, J^{\prime \prime}=40$, (c) $v^{\prime \prime}=0, J^{\prime \prime}=41$, labelled by the probe laser stabilised on the ArI line at $\lambda=5689.9 \AA$. 
which allowed subsequent identification of more complex spectra. Finally, around 900 transitions were assigned to the $C-X$ system (the line positions are available from the authors upon request). The observed long vibrational progressions arise from a large difference in equilibrium distance between the two states, exceeding $20 \%$. The absolute vibrational numbering in the $C$ state has been established unambiguously in our previous experiment [10], taking advantage of the isotope effect in ${ }^{39} \mathrm{~K}^{7} \mathrm{Li}$ and ${ }^{41} \mathrm{~K}^{7} \mathrm{Li}$ molecules.

The measured line positions were fitted to the well-known expansion

$$
v=T^{\prime}\left(v^{\prime}, J^{\prime}\right)-T^{\prime \prime}\left(v^{\prime \prime}, J^{\prime \prime}\right)
$$

with

$$
T(v, J)=T_{\mathrm{e}}+\sum_{i, k}(v+1 / 2)^{i} J^{k}(J+1)^{k} .
$$

As coefficients for the lowest levels of the ground state are known precisely from the previous high resolution experiments $[7,8]$, they were treated as fixed constants in the fits. During the progress of our analysis many determinations of the coefficients have been made for varying amounts of molecular lines and different numbers of Dunham coefficients. The series expansion in Eq. (2) was truncated by ascertaining the degree of the polynomial for which the coefficient of the highest term had a standard error lower than $10 \%$ of its value. The final, best-fit values of the constants, using the largest body of the data (863 lines) are listed in Table II.

\section{TABLE II}

Molecular constants for the $C^{1} \Sigma^{+}$state of ${ }^{39} \mathrm{~K}^{7} \mathrm{Li}$ (in $\mathrm{cm}^{-1}$ ) obtained in this work. The quoted error of a constant is one standard deviation.

\begin{tabular}{l|c|l}
\hline \hline Constant & Value & Error [\%] \\
\hline$T_{\mathrm{e}}$ & 17501.239 & 0.0002 \\
$Y_{10}$ & 115.4135 & 0.014 \\
$Y_{20}$ & -0.152255 & 1.6 \\
$Y_{30} \times 10^{1}$ & -0.127399 & 1.3 \\
$Y_{40} \times 10^{4}$ & 0.59906 & 8.4 \\
$Y_{50} \times 10^{5}$ & -0.10283 & 5.3 \\
$Y_{01}$ & 0.161374 & 0.019 \\
$Y_{11} \times 10^{3}$ & -0.97128 & 0.57 \\
$Y_{21} \times 10^{5}$ & -0.4540 & 8.1 \\
$Y_{31} \times 10^{6}$ & -0.49984 & 1.4 \\
$Y_{02} \times 10^{6}$ & -0.9045 & 0.90 \\
$Y_{12} \times 10^{7}$ & -0.1815 & 2.4
\end{tabular}




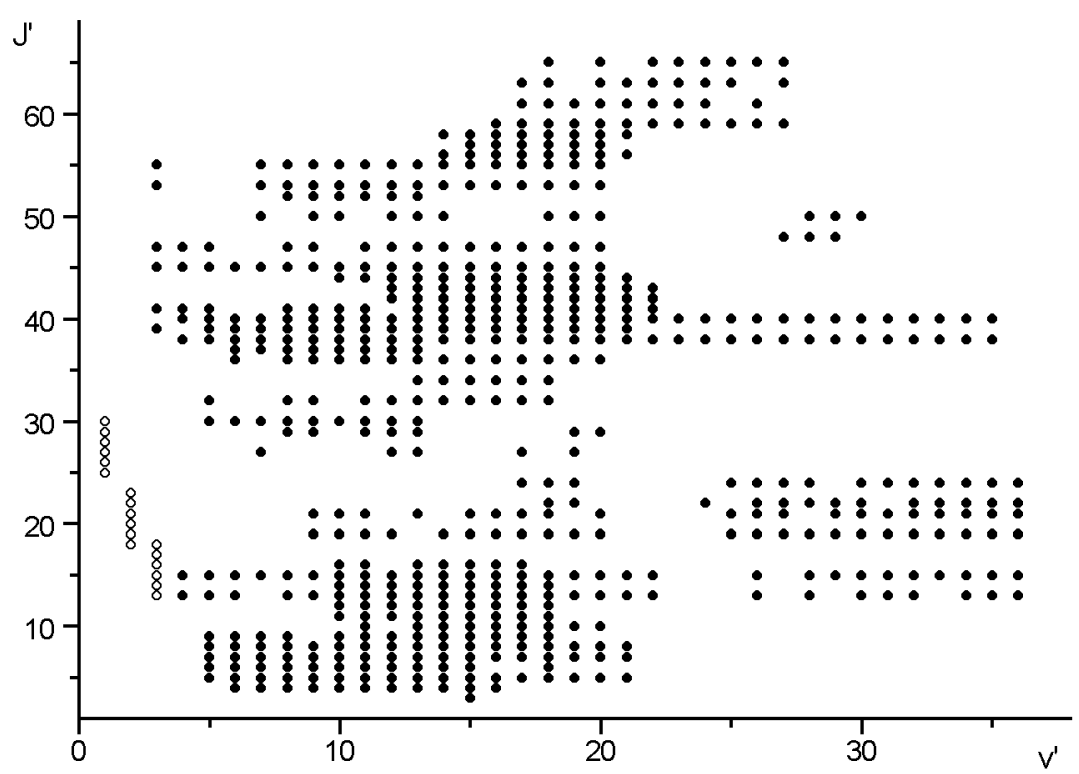

Fig. 2. Range of rovibrational levels of the $C^{1} \Sigma^{+}$state covered in the final fit: solid circles - present measurements, open circles - deperturbed levels $\left(v^{\prime}=1 \div 3\right)$ from our previous high resolution experiment [7].

The root mean square (rms) error of the fit was $0.08 \mathrm{~cm}^{-1}$, which is about the experimental uncertainty. For each constant a total of at least six figures is given, as this number is required to reproduce the measured transitions. Figure 2 the data field used in the final fit which defines the range of applicability of the reported constants. As can be seen, our Dunham coefficients describe the $C^{1} \Sigma^{+}$state from $v^{\prime}=1$ to $v^{\prime}=36$.

Once the coefficients in the Dunham expansion have been determined, we applied the Rydberg-Klein-Rees (RKR) method to obtain the rotationless potential energy curve of the $C^{1} \Sigma^{+}$state. Table III lists some vibrational energy levels and the corresponding classical turning points. It is worth noting that the Dunham constants suggest that there is a small but significant coupling between rotational and vibrational energy in this rather light molecule, shown by the non-negligible value of the zero-point energy correction term, $Y_{00}$, defined as [14]

$$
Y_{00}=\frac{Y_{01}+Y_{20}}{4}-\frac{Y_{11} Y_{10}}{12 Y_{01}}+\frac{Y_{11}^{2} Y_{10}^{2}}{144 Y_{01}^{3}}
$$

(here amounting to $0.081 \mathrm{~cm}^{-1}$ ). Note that $Y_{00}$ is included in the vibrational energies of Table III. The size of this term reflects in this instance the small vibrational anharmonicity of the $C^{1} \Sigma^{+}$state close to its potential minimum. The constant $Y_{20}$ is more than 8 times smaller than in the electronic ground state. 
TABLE III

Rotationless RKR potential for the $C^{1} \Sigma^{+}$state of KLi. The first line refers to the bottom of the potential curve; $R$ is the equilibrium distance.

\begin{tabular}{c|c|c|c}
\hline \hline$v$ & $V\left[\mathrm{~cm}^{-1}\right]$ & $R_{\min }[\AA]$ & $R_{\max }[\AA]$ \\
\hline & 17501.320 & \multicolumn{2}{|c}{4.1917} \\
0 & 559.068 & 3.9798 & 4.4237 \\
1 & 674.136 & 3.8373 & 4.6068 \\
2 & 788.786 & 3.7449 & 4.7399 \\
3 & 902.945 & 3.6733 & 4.8526 \\
4 & 18016.540 & 3.6137 & 4.9537 \\
6 & 241.752 & 3.5170 & 5.1352 \\
8 & 463.859 & 3.4391 & 5.3005 \\
10 & 682.306 & 3.3737 & 5.4566 \\
12 & 896.546 & 3.3172 & 5.6079 \\
14 & 19106.035 & 3.2676 & 5.7570 \\
16 & 310.226 & 3.2236 & 5.9062 \\
18 & 508.566 & 3.1842 & 6.0573 \\
20 & 700.494 & 3.1489 & 6.2120 \\
22 & 885.434 & 3.1171 & 6.3722 \\
24 & 20062.793 & 3.0885 & 6.5396 \\
26 & 231.957 & 3.0628 & 6.7163 \\
28 & 392.287 & 3.0397 & 6.9048 \\
30 & 543.113 & 3.0190 & 7.1080 \\
32 & 683.733 & 3.0005 & 7.3299 \\
34 & 813.408 & 2.9840 & 7.5754 \\
35 & 873.898 & 2.9763 & 7.7091 \\
36 & 931.356 & 2.9689 & 7.8515 \\
& & &
\end{tabular}

The $C^{1} \Sigma^{+}$state is correlated with separated atoms $\mathrm{K}\left(4{ }^{2} S_{1 / 2}\right)+\operatorname{Li}\left(2{ }^{2} P_{1 / 2}\right)$ and its dissociation energy can be obtained from the separation of atomic asymptotes $\operatorname{Li}\left(2^{2} P_{1 / 2}\right)-\operatorname{Li}\left(2{ }^{2} S_{1 / 2}\right)\left(\Delta E=14903.7 \mathrm{~cm}^{-1}[15]\right)$ and dissociation energy of the ground state $\left(D_{\mathrm{e}}\left(X^{1} \Sigma^{+}\right)=6216.4 \mathrm{~cm}^{-1}[9]\right)$, using a standard relation

$$
D_{\mathrm{e}}\left(C^{1} \Sigma^{+}\right)=D_{\mathrm{e}}\left(X^{1} \Sigma^{+}\right)+\Delta E-T_{\mathrm{e}}\left(C^{1} \Sigma^{+}\right) .
$$

This yields the value of $3618.8 \mathrm{~cm}^{-1}$. Thus the Dunham coefficients listed in Table III describe nearly $95 \%$ of the $C$ state potential well depth. Clearly, the low vibrational anharmonicity characterising the bottom of the $C$ state well is quickly lost, as avoided crossings occur between diabatic states of the same symmetry at an increasing internuclear distance. 


\section{TABLE IV}

Comparison of the experimental and theoretical molecular constants of the $C^{1} \Sigma^{+}$state in KLi. All values are in $\mathrm{cm}^{-1}$, except for $R_{\mathrm{e}}$ in $\AA$.

\begin{tabular}{c|c|c}
\hline \hline Constant & Experiment (this work) & Theory [16] \\
\hline$T_{\mathrm{e}}$ & 17501.239 & 17647 \\
$\omega_{\mathrm{e}}$ & 115.41 & 114.27 \\
$B_{\mathrm{e}}$ & 0.161374 & 0.16494 \\
$D_{\mathrm{e}}$ & 3618.8 & 3477 \\
$R_{\mathrm{e}}$ & 4.192 & 4.128
\end{tabular}

It is interesting to compare the experimentally obtained parameters of the $C$ state with the results of most recent theoretical calculations [16] (see Table IV). It can be seen that the ab initio treatment places the bottom of the $C^{1} \Sigma^{+}$state by some $150 \mathrm{~cm}^{-1}$ too high, and slightly underestimates the values of $\omega_{e}, R_{e}$, and $D_{e}$, but the discrepancies do not exceed $4 \%$ of the experimental values. This illustrates the performance of modern quantum chemistry in predicting properties of yet unobserved molecular states in diatomic molecules.

\section{Acknowledgments}

A.G., P.K. and W.J. gratefully acknowledge financial support from the State Committee for Scientific Research (grant No. 2 P03B 063 23). This collaboration also received funding from the France-Poland exchange programme "Polonium" and from a "Convention d'Echange" between the CNRS in France and the Polish Academy of Sciences.

\section{References}

[1] S. Magnier, M. Aubert-Frécon, Ph. Millié, J. Mol. Spectrosc. 200, 96 (2000).

[2] S. Rousseau, A.R. Allouche, M. Aubert-Frécon, J. Mol. Spectrosc. 203, 235 (2000).

[3] H. Fahs, A.R. Allouche, M. Korek, M. Aubert-Frécon, J. Phys. B 35, 1501 (2002).

[4] R.F. Barrow, P. Crozet, Ann. Rep. Prog. Chem. C 89, 353 (1992).

[5] V. Bednarska, I. Jackowska W. Jastrzębski, P. Kowalczyk, Meas. Sci. Technol. 7, 1291 (1996).

[6] V. Bednarska, P. Kowalczyk, W. Jastrzębski, J. Mol. Spectrosc. 180, 435 (1996).

[7] V. Bednarska, A. Ekers, P. Kowalczyk, W. Jastrzębski, J. Chem. Phys. 106, 6332 (1997).

[8] W. Jastrzębski, P. Kowalczyk, Spectrochimica Acta A 54, 459 (1998).

[9] F. Martin, P. Crozet, A.J. Ross, M. Aubert-Frécon, P. Kowalczyk, W. Jastrzębski, A. Pashov, J. Chem. Phys. 115, 4118 (2001). 
[10] A. Pashov, W. Jastrzębski, P. Kowalczyk, Chem. Phys. Lett. 292, 615 (1998).

[11] W. Jastrzębski, P. Kowalczyk, A. Pashov, J. Mol. Spectrosc. 209, 50 (2001).

[12] M.G. Littman, H.J. Metcalf, Appl. Opt. 17, 2224 (1978).

[13] R. Ferber, W. Jastrzębski, P. Kowalczyk, J. Quant. Spectrosc. Radiat. Transfer 58, 53 (1997).

[14] G. Herzberg, Molecular Spectra and Molecular Structure, Vol. 1: Spectra of Diatomic Molecules, Van Nostrand, Princeton 1950, p. 109.

[15] A.A. Radzig, B.M. Sminnov, Reference Data on Atoms, Molecules and Ions, Springer-Verlag, Berlin 1985, p. 149.

[16] S. Rousseau, A.R. Allouche, M. Aubert-Frécon, S. Magnier, P. Kowalczyk, W. Jastrzębski, Chem. Phys. 247, 193 (1999). 